\title{
Promotion of improved rice parboiling technology in the Innovation Platforms in Sierra Leone
}

\author{
Kemoh Bangura',2, Nazir A. Mahmood ${ }^{1}$, Abdulai Bangura ${ }^{1}$ and Jean Moreira ${ }^{3}$ \\ 1. Rokupr Agricultural Research Center (RARC)/Sierra Leone Agricultural Research Institute (SLARI) \\ 2. Department of Agricultural and Biosystems Engineering, Njala University, Sierra Leone \\ 3. Africa Rice Center (AfricaRice), Cotonou
}

\begin{abstract}
In this study, 20 local artisans were trained on the fabrication of improved rice parboiling technology. 100 women and 50 men were trained on the use of the improved parboiling technology. Participants were selected from female and male farmers from different farmer-based organizations. The scaling-out of this technology to the rice sector development hubs in Sierra Leone will reduce the constraints related to the traditional parboiling process and improve the productivity and quality of the parboil rice in conformity with the market demand. It will also help to increase the income of the actors along the rice value chain.
\end{abstract}

Keywords: Improved rice parboiler, fabrication, training, rice hubs.

\section{Introduction}

Rice is the staple food for the Sierra Leonean population. The average rice consumption per person during the year varies between 80 to $104 \mathrm{~kg}$ of net rice. About $80 \%$ of the local rice consume in Sierra Leone is parboil rice particularly in the North. But it is important to note that traditional rice parboiling methods in pots and half cut barrels on three stone stove with less capacity (10 to $15 \mathrm{~kg}$ of paddy rice) are mostly used. However, there are several challenging issues related to the use of the traditional rice parboiling methods, such as high use of fuel (firewood), high consumption of water during the heating or precooking of the paddy, long parboiling time, low quality of the rice produce from this process, poor cooking quality etc.)

The objective of this study being to generate and speed up the diffusion and adoption of improve parboiling technology under low cost in order to reduce the challenges related to the traditional rice parboiling process, increase the rice outputs, meets the needs of the market and increase the income of the actors in the rice sector in Sierra Leone.

\section{Materials and methods}

In an effort to diffuse the improved paddy rice parboiling technology in Sierra Leone, 20 local artisans were trained on the fabrication of the improved paddy rice parboiler. Next, 100 women and 50 men were trained on the use of the improved rice parboiling technology. Participants were selected from female and male farmers from different farmerbased organizations (FBOs) in the two main rice hubs, the Mangrove and the Inland Valley Swamp respectively. These FBOs identified their members whom they think can adequately conduct training of trainers within their communities.
Results

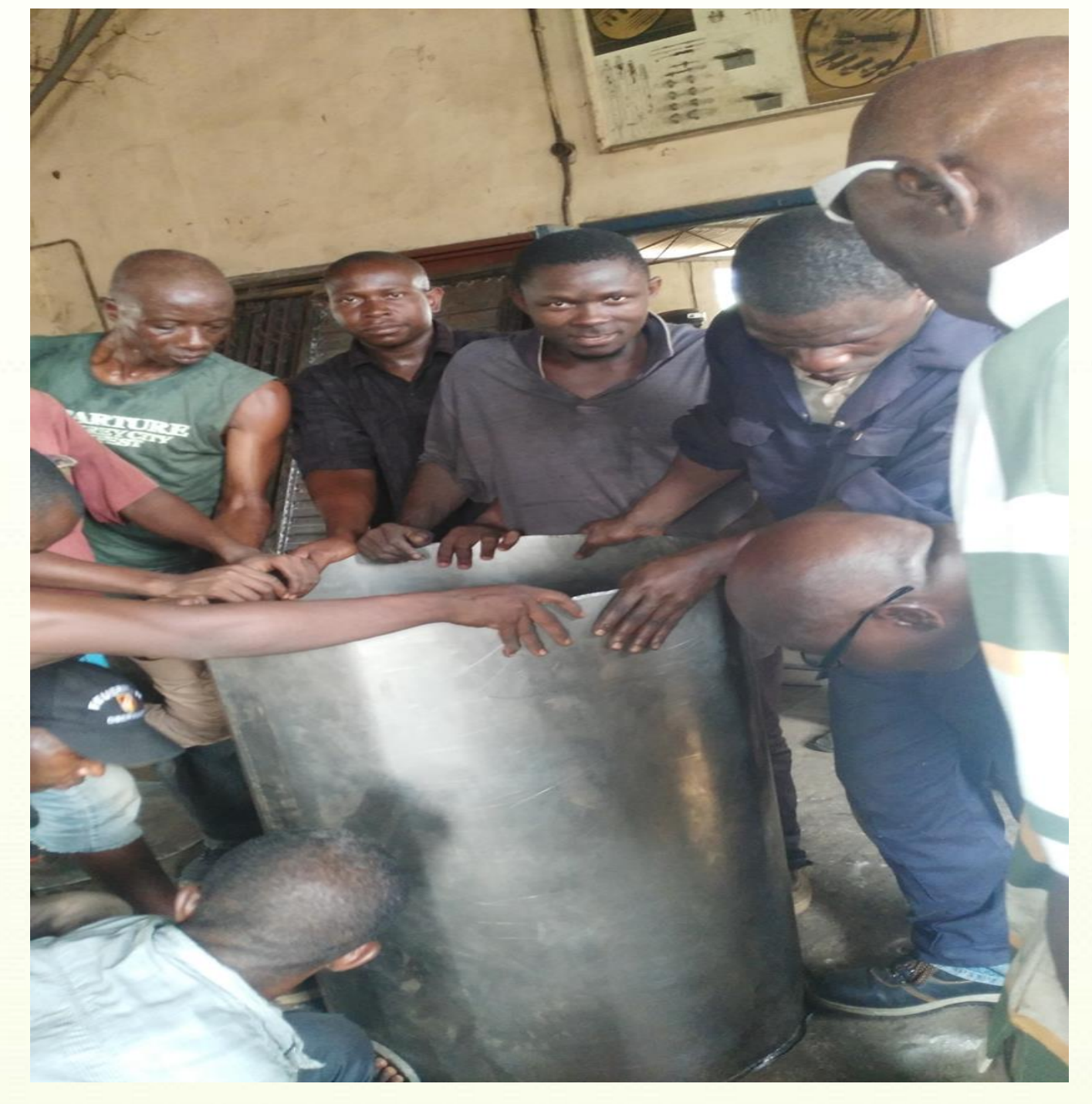

Training on the fabrication of the improved rice parboiler

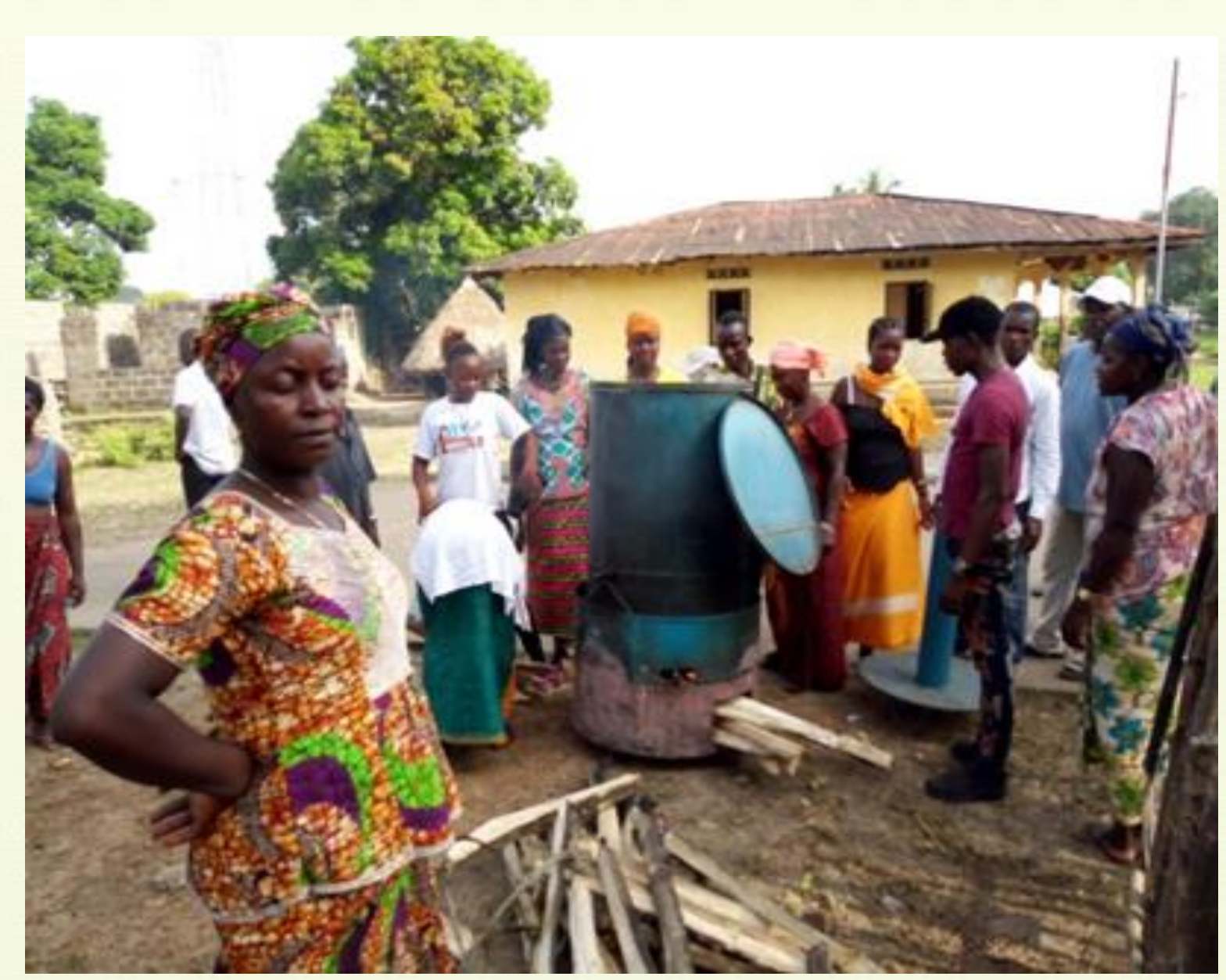

Farmers training on the use of the improved rice parboiler

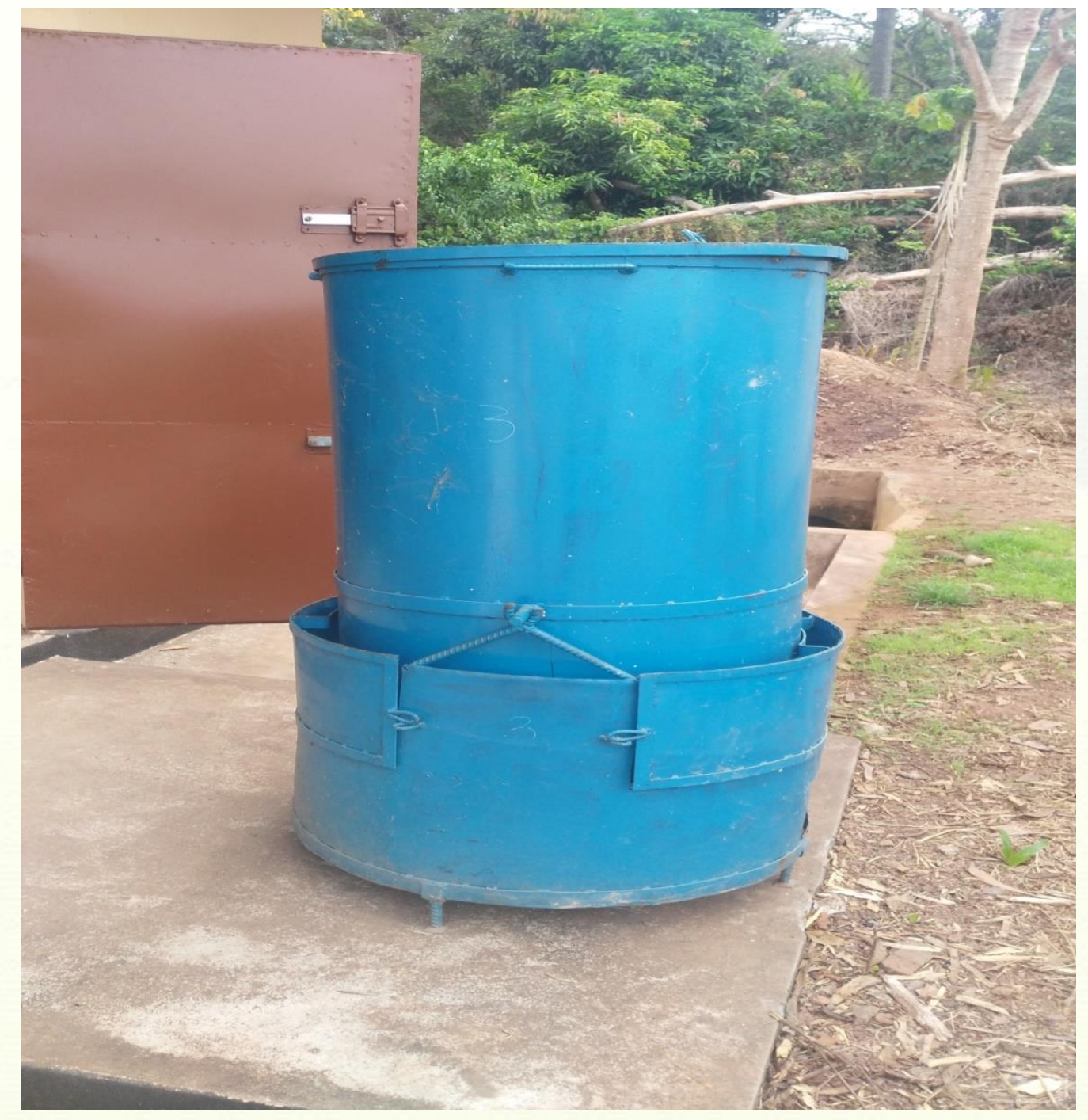

The Improved rice parboiler

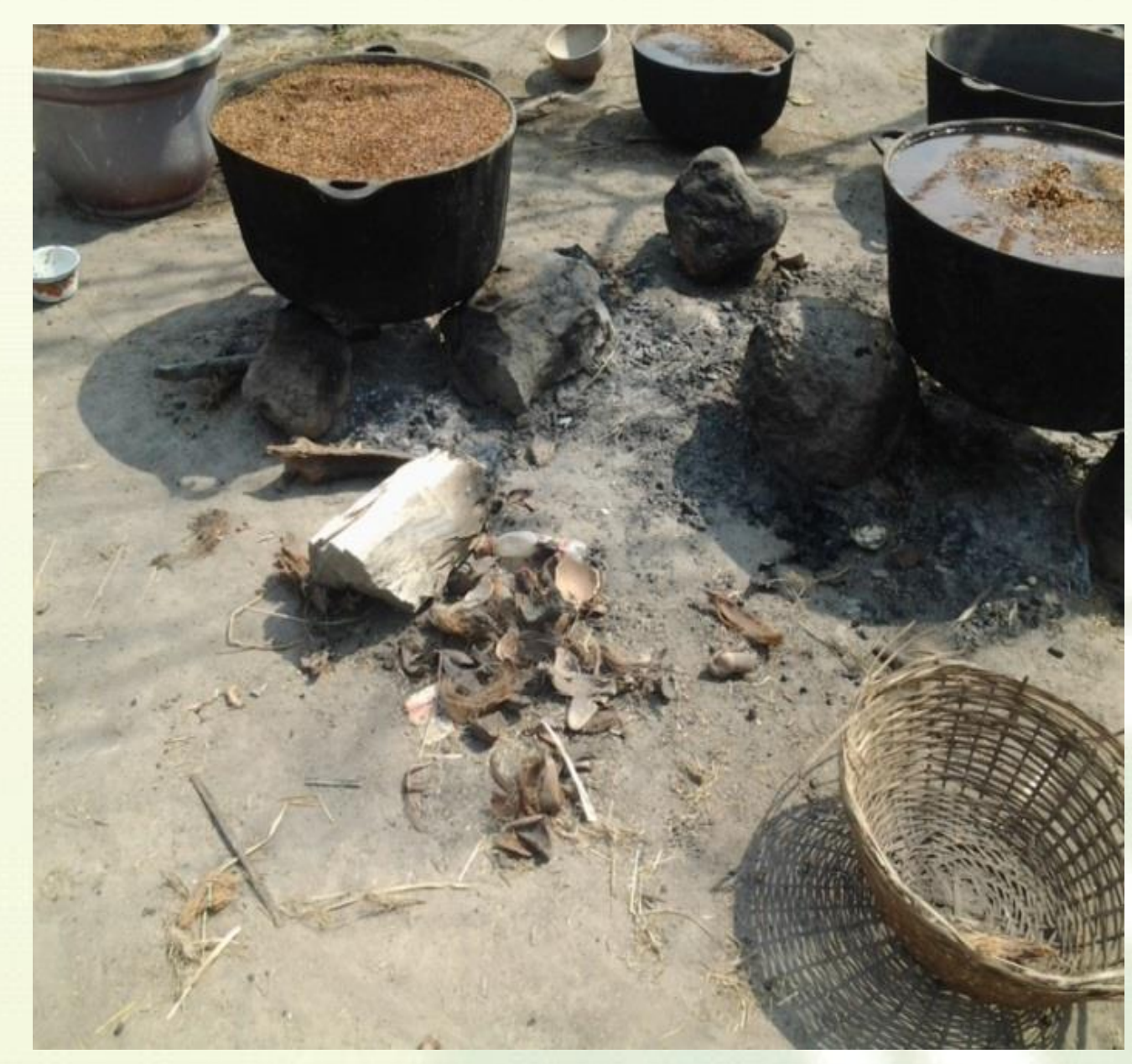

Traditional parboiling of rice in Sierra Leone using pots and half cut barrels.

\section{Conclusions}

The participants were able to understand and appreciate the use of the improved rice parboiling technology. They were able to compare the improved paddy rice parboiler to the traditional parboiling methods. The improve rice parboiler was found to be efficient in improving the quality of the parboiled rice, reduce firewood consumption and reduce time spent during parboiling. It is expected that the scaling-out of this technology to the rice sector development hubs in Sierra Leone will enhance the timely execution of the rice parboiling operation, substantially reduce postharvest losses, and improve grain quality while freeing up family labour for other activities. Higher grain quality is a prerequisite for competitiveness of local rice against imports that cost Sierra Leone much valuable foreign exchange. The wide dissemination of the equipment will facilitate the involvement of the private sector operators in rice value chain activities. It will also help to create employment for the youths and women.

\section{Future research direction}

Scaling-out of the improved rice parboiling technology to the rice sector development hubs in Sierra Leone. 